\title{
Cyborg art y bioética: Stelarc y The third ear
}

\section{Cyborg Art and Bioethics: Stelarc and The Third Ear}

\author{
Valeria Radrigán \\ Universidad de Valparaiso \\ valradrigan@yahoo.es
}

\section{Resumen:}

El texto revisa las posibilidades del cuerpo como estructura unitaria y su reconfiguración a través del desarrollo tecnológico. Se realiza un informe sobre el estado de la cuestión, considerando que la rearticulación conceptual y física de lo humano, tiene su grado más radical en el contexto artístico, donde aparecen en tensión preguntas por la noción de límite en el cuerpo y su conflicitividad ética. Considerando que estas prácticas (sobre todo en el contexto latinoamericano) son emergentes, se hace necesario desarrollar este tema revisando la tradición artística internacional como análisis de caso. En concreto, la obra del australiano Stelarc, permitirá establecer un buen correlato crítico del problema, analizando cómo estas modificaciones corporales conllevan nuevos sistemas de representación, manejos de la identidad, etc.

Palabras clave: cyborg art, bioética, cuerpo, transhumanismo, perfomatividad.

\begin{abstract}
:
This paper looks into the possibilities of the body as a unitary structure and its reconfiguration through technological development. It includes a report on the state of the question, considering that conceptual and physical re-articulation of the human reaches its most radical degree within the context of art, where questions about the notion of limit on the body and ethical conflicts emerge. Considering that these practices (particularly in the Latin American context) are recent, it is necessary to develop this subject by reviewing the international artistic tradition. Particularly, the work of the Australian Stelarc will set a good example of the problem, analyzing how these body modifications involve new systems of representation, identity handling, etc.
\end{abstract}

Keywords: Cyborg Art, Bioethics, Body, Transhumanism, Performativity. 
En la actualidad, dentro de la escena cultural y social, nos encontramos con acciones que trabajan en/con el cuerpo y las nuevas tecnologías a través de estrategias que incluyen la intervención directa de la carne y la manipulación de las estructuras biológicas básicas. Procedimientos como la manipulación genética, la cirugía, la implantación de prótesis, etc., nos hacen cuestionar directa o indirectamente los límites del cuerpo humano así como lo conocemos.

La actividad artística contemporánea no está ajena a estas problemáticas, proponiendo, desde su propia institucionalidad, una crítica activa en el marco de las prácticas más radicales en cuanto al grado de determinación y sujeción de lo vivo. Dentro de estas, existe una gama de acciones que proponen confrontar y exponer las tensiones propias del cuerpo y su configuración, exponiendo un nudo de debates en el que se cruzan los límites éticos sobre la experimentación con seres humanos, la autogestión del cuerpo y la posibilidad de trascender todo determinismo tecnológico. En este deslinde disciplinar y conceptual, aparece de fondo la posibilidad de repensar nuestra condición de especie en el mundo, trascendiendo estructuras mentales muy profundas como el dualismo que ha determinado el pensamiento occidental desde el renacimiento.

En este contexto, intentaremos exponer cómo la tradición del cyborg art a través de la figura del artista australiano Stelarc, instala una nueva mirada y articulación simbólica sobre la (ciber)cultura, poniendo en crisis tanto el modo en que percibimos las estructuras corpóreas, como la posibilidad de mediar performativamente nuestra propia carnalidad. Veremos de qué modo esta estrategia artística promueve un discurso de reivindicación y obsolescencia del cuerpo orgánico en virtud de las tensiones físicas y culturales a las que este se enfrenta mediado por las tecnologías. En el cyborg art, el cuerpo como unidad biológica, como construcción cultural y como soporte de identidad está puesto en cuestión, reconfigurando el campo de las artes vivas ${ }^{1}$ e instalando una necesaria discusión sobre las implicancias éticas del desarrollo científico-tecnológico en el campo de lo vivo, ámbito de acción propio de la bioética: "Desde aquí ya se puede asumir que la bioética se localiza en la encrucijada contemporánea donde convergen y se problematizan dificultades de índole moral derivadas de la intervención técnica sobre la vida (en sus múltiples expresiones) y el medio ambiente que de manera global la sustenta" (Villarroel 145).

Particularmente, Stelarc ha desarrollado un grueso de obra basándose en la premisa de que el cuerpo está obsoleto. Su obra se centra en las posibilidades de extensión y modificación del cuerpo humano a través de la tecnología, para lo cual ha investigado en medicina, robótica, mecanismos de control remoto del cuerpo, etc. Esta producción resulta particularmente relevante, pues nos conduce a una región fronteriza, en donde los límites de lo éticamente aceptado en cuanto a la intervención y manipulación del cuerpo, son transgredidos, cuestionados y/o reafirmados. De este modo, revisaremos cómo sus creaciones de mutaciones antropo-máquinicas - particularmente la obra The third ear-nos

1 Tomado del inglés live art (francés: arts vivants) para referirse al amplio espectro de las artes que tienen como centro la figura del cuerpo humano. Se incluirían, por tanto, las artes escénicas, el arte de acción, performance, etc. 
inducen al cuestionamiento por el presente y futuro de nuestra especie, así como por el estado actual y proyectivo de la praxis artística. Con respecto a esta última, dirigiremos el análisis hacia la condición privilegiada del arte (compartida con la ciencia) como espacio de exención ética con respecto a prácticas y métodos que involucran la intervención, manipulación y reformulación de lo humano.

\section{Transgresión del cuerpo - transgresión ética}

Debemos recordar que el cuerpo, más allá de cualquier postura filosófica o antropológica, es nuestro soporte de acción y relación con el mundo. El mayor o menor grado de identificación de esta unidad con la totalidad de lo humano, o la valorización o sacralización de esta estructura, varían, por cierto, según autores, culturas y tiempos, pero es claro que parece existir una suerte de consenso en relación al respeto y cuidado que debemos tener con la corporalidad: "Cada cultura humaniza la carnalidad, al mismo tiempo que utiliza el cuerpo como representante de una ética y una estética” (Ciénaga). Desde esta perspectiva, adulterar nuestro cuerpo resulta un tabú cultural: "las fronteras del cuerpo, que son simultáneamente los límites identitarios del individuo mismo, saltan en pedazos y siembran el descontrol" (Le Breton 29).

Efectivamente, la naturaleza opera como sistema de modificación y mutación constante, permitiendo el cambio sucesivo de estructuras corporales tanto humanas, como vegetales o animales. Sin embargo, cuando las alteraciones surgen como impulsos o voluntades propias de los sujetos, el traspaso de límites opera en un cierto rango de admisibilidad menor que el de las mutaciones producto del medio. Modificar la especie o alterar la vida tendrían como resultado, según diversas mitologías y discursos culturales, una catástrofe que tendería, eventualmente, a nuestra destrucción. Pareciera ser que toda instancia que tienda hacia el descontrol, la hibridez o indiferenciación de lo humano, nos sitúa frente a una sensación de caos muy primitiva, que demanda en consecuencia un ordenamiento base para poder habitar el mundo. Si el hombre tiende, de forma consciente, a anular estas estructuras de orden y diferenciación promoviendo una alteración definitiva de su estructura primera, se difuminan en consecuencia las leyes básicas -o la ética más profunda- de una comunidad.

Resulta imposible no rastrear el germen de este cierre corporal a un nivel epistemológico y en la filosofía occidental, a través de las figuras de Platón y Descartes. Si bien estos pensadores se sitúan desde diversos contextos y con distintos enfoques, ambos son exponentes fundamentales de la tradición dualista que heredamos, mediante la cual entendemos la realidad a partir de oposiciones como cuerpo-alma (mente) o sujeto-naturaleza, por ejemplo. Recordemos que Platón propone una visión del cuerpo en la que este es un material mortal corruptible y propio del mundo sensible, por lo que se presenta como estorbo para el alma (inmaterial e inmortal) en su acceso a las ideas. En el caso de Descartes, su propuesta en torno a la res cogitans (mente, aparato racional) se establece como oposición y superación a la res extensa (cuerpo orgánico), que aparece 
además como un entramado carnal de músculos y huesos susceptible a podredumbre y descomposición. A su vez, este pensamiento se encuentra inscrito en el seno de la investigación científico-experimental que permitió la disección y estudio del cuerpo a través de los cadáveres, cuestión que facilitó una verdadera y literal apertura de la carne para definir las cualidades de la vida a partir de la muerte: "hermosa transmutación del cadáver: un tierno respeto lo condenaba a pudrirse, al trabajo negro de la destrucción; en la intrepidez del gesto que no viola sino para sacar a luz, el cadáver se convierte en el momento más claro en los rostros de la verdad. El saber prosigue donde se formaba la larva." (Foucault 178). Con ello, la vida empieza a definirse desde una actitud limítrofe y como una oposición a la muerte, como una suerte de fondo de existencia solo perceptible a través del ejercicio de la dualidad.

En este marco, la definición y valoración de lo humano aparece claramente mediada por un determinismo científico, que desde la superioridad del pensamiento racional es capaz de diseccionar y clasificar el cuerpo orgánico en un afán de total comprensión. Al mismo tiempo, las representaciones visuales asociadas a lo humano a partir de este momento, heredan cánones propios de los atlas anatómicos, con lo que el cuerpo acaba por convertirse -a pesar de Descartes- en un eje fundamental en la percepción de nuestra especie. Con esto llegamos a que las modificaciones radicales que se realicen sobre/en el cuerpo tendrán como consecuencia también un traspaso de límites políticos clave: ¿hasta qué punto tienen las instituciones -en particular la científica- poder para determinar nuestro control corporal? ¿Puede y tiene derecho el arte de atentar contra estas regulaciones?

Es aquí donde el entramado se vincula con las problemáticas de índole ética que hemos esbozado antes, pues en rigor y más allá de toda institucionalidad, ¿hasta dónde es correcto intervenir la corporalidad? ¿La sustitución, modificación o amputación voluntaria de partes del cuerpo es algo que beneficie al sujeto o al contrario, algo que puede atentar contra su destrucción? Instalaremos como premisa de trabajo, que la actividad artística no puede operar con esta exención ética o autonomía total, partiendo por el hecho de que tanto la ética como la estética operan como valores para una comunidad, por lo que se encuentran intrínsecamente unidas. Recordemos que la ética en cuanto ethos se entiende como carácter, costumbre o hábito y también como morada; tres aspectos relacionados con una experiencia interior del sujeto pero tramado por la cultura en tanto el hábito se genera por repetición en un determinado contexto cultural $^{2}$.

2 Ricardo Maliandi (filósofo argentino, especializado en ética), entre otros autores, entrega antecedentes sobre el origen etimológico del ethos. Volviendo a su origen griego y señala la diferenciación entre êthos y éthos, puntualizando que si bien ambos pueden traducirse como costumbre, el primero tiene una mayor connotación moral, traduciéndolo como carácter, como lo más propio de una persona y de su forma de actuar. Mientras que el segundo haría alusión al hábito, aunque reconoce que semánticamente estarían relacionados (como habría quedado de manifiesto en la propia filosofía griega), una de las vinculaciones sugeridas es justamente la de que el carácter se forma a través del hábito. A su vez, agrega como antecedente que el mismo êthos tenía en el griego clásico una acepción más antigua referida a vivienda, morada, lugar donde se habita, rescatando su nexo con las otras dos acepciones en el hecho de aludir a lo "propio, lo íntimo, lo endógeno: aquello de donde se sale y a donde se vuelve, o bien aquello de donde salen los propios actos, la fuente de tales actos" (Maliandi 13-4). 
Si además consideramos como preocupación fundamental de la ética los actos humanos que se realizan tanto por la voluntad como por la libertad absoluta de la persona, podemos encontrar un profundo vínculo con la actividad artística en el mundo contemporáneo. Además, el entendimiento del mundo desde una perspectiva ética, solo puede sustentarse en una percepción estética, entendida esta como una comprensión de la sensibilidad en el marco de una comunidad establecida. Sin una capacidad de distinguir intrínsecamente el placer del displacer, será imposible a posteriori adquirir conciencia de lo que se percibe bajo las normativas del grupo al que se pertenece. Por estas razones, lo ético-estético es algo que pertenece al sujeto como parte de su estructura perceptiva y representativa, la cual se sustenta en la comunidad y contexto histórico en la que desarrolló sus facultades mentales, emocionales, etc. Si bien Hegel en su Fenomenología del espíritu define la eticidad como una existencia previa a la cultura, como una experiencia personal o del espíritu, lo cierto es que al mismo tiempo señala que una buena conciencia actúa y obra en un ser para otro, con lo que es en la interacción humana donde se despliega la ética como universalidad.

Entendemos entonces que todo arte y toda experiencia estética está inserta en una sociedad que se rige por normas básicas de convivencia para su organización y operatividad. Si bien uno de los grandes motores del arte -y del arte contemporáneo en particular- resulta de la crítica y puesta en tensión de la cultura, debiéramos al menos instalar la discusión sobre los límites aceptables de esta crisis. Ya Kant en su Crítica del juicio nos advertía de esta problemática, preguntándose por el lugar de la voluntad en un mundo determinado por leyes. En este marco, discute por la posibilidad de la belleza y la experiencia estética dentro de estas leyes que todo lo rigen. ¿Es que lo bello se produce y percibe también desde una norma? Para Kant, existe la posibilidad de que una obra de arte nos permita trascender el puro raciocinio de la ley para ingresar en un momento de pura experiencia o armonía cósmica, donde parecieran encontrarse entendimiento y sensibilidad. Este instante, que va más allá del entendimiento, refresca el mundo, rearticulando la relación con él y con las leyes que, de forma categórica, lo sustentan. Al ingresar en este territorio donde operaría una libre integración de facultades, los conceptos asociados a los objetos u experiencias son trascendidos por la experiencia, provocándose una sensación de novedad que nos permite mirar nuestro entorno con otros ojos ${ }^{3}$.

Sería entonces un nuevo mirar hacia la corporalidad lo que estaría propuesto a través de las obras de cyborg art, que pese a estar inscritas en un régimen de producción y visualidad completamente distintos a la propuesta kantiana nos permiten articular una experiencia libre y a la vez crítica de nuestra realidad.

3 Si bien Kant y su propuesta opera en un régimen de la visión completamente distinto al del arte contemporáneo y en particular a las estrategias del cyborg art, sí nos interesa rescatar la idea de la rearticulación y refrescamiento de la mirada que creemos está presente como voluntad artística particularmente en las obras de Stelarc. 


\section{The third ear: el cuerpo como expansión tecnológica}

En la producción artística de Stelarc, vemos que él actúa libre, crítica y conscientemente sobre su propio cuerpo, operando en lo que Bajtín ${ }^{4}$ denominaría un acto responsable, éticamente correcto:

la responsabilidad ética se concentra en cada texto-acción-obra artística, a la vez que se
distribuye históricamente. De este modo, la creatividad artística, la historia- particular-
mente el arte y la cultura-, la crítica y, [...] la estética y la ética, reafirman su carácter
de disciplinas abiertas que en permanente interacción van completando la mirada,
haciendo otras lecturas y reescribiendo responsablemente nuevos textos (Cimaomo).

Desde esta perspectiva, las acciones de Stelarc son responsables en la medida en que "asumen la singularidad de sus enunciados- dando cuenta desde su lenguaje y óptica particular de una determinada conciencia histórica" (Cimaomo). En esta misma línea, podemos decir que operan en lo que la bioética ha llamado consentimiento informado: "[...] la adhesión libre y racional del sujeto a un procedimiento propuesto por el equipo de salud, sea con intención diagnóstica, pronóstica, terapéutica o experimental, e incluye competencia, información y libertad" (Manzini 40). Con esto, no debiera mediar aparentemente problemática ética alguna. Sin embargo, si como hemos visto el cuerpo es un eje determinante en la definición y valoración de lo humano, ¿hasta dónde es posible tensionar esta estructura sin transgredir parámetros (bio)éticos básicos? ¿Puede el consentimiento informado mediar como única norma de control ante la propia manipulación del cuerpo? Intentaremos abordar estas preguntas a través de la obra The third ear de Stelarc, inscribiéndola en la tradición del cyborg art y poniéndola en tensión con conceptos clave para la relación (bio)ética y estética.

Esta obra, que comenzó a desarrollarse en 2007, cuestiona fuertemente las fronteras de lo humano y la posibilidad de expansión y actualización del cuerpo a través de la tecnología. En este caso, la obra consiste en el injerto quirúrgico de una falsa oreja en el brazo del artista que a su vez busca ser un dispositivo de comunicación, poseyendo un sensor de proximidad que detecta a personas que se acercan a ella, una microantena $w i-f i$ y un pequeño micrófono que permite recoger los sonidos del exterior. El diseño y modelación de la oreja fue elaborado en 3D por el Spatial Information Architecture Lab de Melbourne y el proceso de implante y postoperatorio de la oreja, realizado por un equipo de médicos europeos calificados.

La radicalidad de esta performance opera en varios niveles. En primer lugar, su consentimiento informado trasciende el saber las consecuencias de la operación en un ámbito estrictamente de salud: él participa activamente del diseño y el proceso quirúrgico

4 El desarrollo de esta propuesta se encuentra en Bajtín, M. Hacia una filosofía del acto ético. San Juan: Universidad de Puerto Rico, 1997. Medio impreso. 
mediante una opinión crítica y política con respecto a la autogestión del cuerpo. En segundo lugar, la obra claramente atenta contra toda estructura normal del cuerpo perturbándolo a través de la modificación permanente de sus partes. Hay, mediante un acto de cirugía, un re-tratamiento y re-consideración de este mismo procedimiento no para curar o corregir en el sentido médico tradicional, sino para cuestionar justamente las nociones de cura y corrección. Nadie necesita, desde la operacionalidad o estética social tener una oreja en el brazo (de hecho esto parece anti-funcional y anti-estético), sin embargo Stelarc se pregunta por esto mismo en su sitio web oficial:

I have always been intrigued about engineering a soft prosthesis using my own skin, as a permanent modification of the body architecture. The assumption being that if the body was altered it might mean adjusting its awareness. Engineering an alternate anatomical architecture, one that also performs telematically. Certainly what becomes important now is not merely the body's identity, but its connectivity- not its mobility or location, but its interface. In these projects and performances, a prosthesis is not seen as a sign of lack but rather as a symptom of excess. As technology proliferates and microminiaturizes it becomes biocompatible in both scale and substance and is incorporated as a component of the body. These prosthetic attachments and implants are not simply replacements for a part of the body that has been traumatized or has been amputated. These are prosthetic objects that augment the body's architecture, engineering extended operational systems of bodies and bits of bodies, spatially separated but electronically connected (Stelarc, "Ear on Arm”).

La idea de considerar al cuerpo como una interfaz, o la posibilidad de entender la prótesis como una parte extensiva del cuerpo (no como un reemplazo o sustitución de algo defectuoso sino como un aumento de las posibilidades orgánicas) sitúan claramente a la obra en la tradición del cyborg art. Aquí debemos recordar que este tipo de estrategias se sustentan en la premisa del posthumanismo, corriente que postula que el ser humano como especie se encuentra en un estado de modificación radical de su corporalidad en virtud de las relaciones que se establecen con la tecnología. Dicha propuesta, leída desde la caída de las grandes utopías modernas con respecto a la ciencia, tiene claramente una lectura de tinte apocalíptico: los avances tecnológicos no solo han acabado con nuestro entorno sino que amenazan radicalmente con la extensión de nuestra especie. "La creciente intervención del hombre en el curso de la naturaleza y, al mismo tiempo, en su propia naturaleza, hace incontestable que el poder del hombre puede afirmarse eminentemente como poder de destrucción del mundo del hombre y desnaturalización del hombre mismo" (Stiegler 141). La figura del cyborg (cybernetic organism, híbrido entre máquina y hombre) aparece aquí como un ícono del terror, reflejada en personajes como Frankenstein o Terminator.

Sin embargo, teóricos como Haraway y Hayles postularán una mirada de reivindicación para el cyborg, considerándolo una creatura híbrida integradora de opuestos, superadora de dicotomías e, incluso, subversiva para con la tecnología, desde la perspectiva 
de que pudiera surgir como imagen-deseo de un sujeto que, desde su voluntad crítica, opta por trascender las limitaciones de su físico gracias a los avances de la ciencia: "I am not my biology. I will be the one to decide what my body grows into" (Larratt 7).

Lo interesante de esta figura, es que opera como representación de la ciencia ficción pero también de la realidad de la ciencia en el mundo contemporáneo, factor que ha sido en efecto de influencia para artistas como Stelarc que optan por asumir esta mirada de reivindicación cibernética en su arte. Está de fondo un profundo "cuestionamiento sobre la forma del cuerpo y su construcción, destrucción y alteración por los sistemas de poder y por una historia artificial- la medicina, la cultura, los gobiernos, los procesos que más allá de su naturaleza biológica articulaban la imagen corporal." (Cimaomo). Este análisis trae como consecuencia directa el deseo de confrontar estas estructuras rígidas que daban forma y normaban las concepciones sobre el cuerpo, tomando las riendas de la situación y decidiendo obrar sobre el propio modificándolo de forma radical, a voluntad. La figura del cyborg/artista -y de Stelarc en particular, podríamos decir- es aquí la de un artista soberano, que trasciende toda concepción o noción de un cuerpo sacro inalterable para asumir, desde un antropocentrismo total, el dominio de su propia estructura gracias a las tecnologías que, aparentemente, dominaban y moldeaban al humano como especie.

¿Es posible ejercer esta soberanía de forma total? Aparentemente, el artista actúa aquí como completamente desvinculado de la sociedad en la que se inserta. Se sitúa como ser autosuficiente con el poder de hacer lo que quiera sobre sí mismo, sin que esto tenga consecuencia alguna en su medio y olvidando que el que una comunidad establezca ciertos patrones de valoración o conducta, no es un ejercicio gratuito, sino producto de una serie de factores que, en el tiempo se concretizan de forma colectiva. Aquí resulta importante nuevamente aludir al tema de la conciencia crítica y el acto responsable, ámbitos que, como expresamos antes, están profundamente vinculados. Stelarc, inscrito en la tradición del cyborg art y exponente clave de esta tendencia, es un artista que ha estudiado y dedicado su vida profesional al estudio del cuerpo, sus modificaciones y potencialidades de expresión. Al mismo tiempo, trabaja en conjunto con un equipo transdisciplinario a través del cual se nutre en conocimientos, técnicas y estrategias que le permiten sustentar y realizar sus propuestas. Amparado tanto en la institución arte como en la institución ciencia, realiza sus obras con el objetivo de modificar paradigmas y estructuras de pensamiento asociadas a la relación cuerpo y tecnología, acercándolas a cambios efectivos que él percibe en la cotidianidad actual. No olvidemos en este contexto la conocida premisa de Donna Haraway: "todos somos cyborgs", inscrita ya en el Manifiesto Cyborg de 1985. Casi veinte años han pasado ya desde esta aseveración y cada vez se hace más patente que:

Los sujetos han incorporado a su vida cotidiana una diversidad de aparatos protésicos que se han vuelto imprescindibles: teléfonos móviles, ordenadores portátiles, miniordenadores, palm pilot, mp3, iPod, iPhone, reproductores de CD y DVD, cámaras de fotografía y filmación digitales, etc... dispositivos por doquier que crean realidades 
intangibles. E-mails, blogs, facebook, myspace, youtube, skype, google, compras y pagos de cuentas on-line, mensajes de texto general otras formas de comunicación y otra clase de vínculos (Cotaimich).

Con lo que resulta evidente que nuestra realidad cotidiana demanda una revisión de los patrones sociales que definen el cuerpo como unidad fija, inmutable, inmodificable. Más aun, se requiere una mirada crítica sobre la tecnología que trascienda los debates apocalípticos y devuelva al sujeto la posibilidad de un accionar consciente para con las tecnologías que lo rodean. Ahora: ¿es esto acaso factible? Autores como Vilem Flusser en Hacia una filosofía de la fotografía, plantean una imposibilidad de accionar con libertad frente a aparatos programados que, de hecho, nos estarían programando a nosotros. La autonomía del sujeto y su capacidad de discernir se verían mermadas o incluso anuladas por los dispositivos y los mecanismos de control que los trascienden, a lo cual Flusser llama el metaprograma. De este modo, lo humano se encontraría en crisis o en estado de anulación, cuestión que desde la sociología también ha sido abordada por Lewis Mumford, quien plantea en Técnica y civilización el concepto de la megamáquina como metasistema que subsume al hombre en un estado de esclavitud total frente a la tecnología.

En este contexto, solo las frías y utilitarias lógicas de la ciencia dominan toda posible vida: el calor de un abrazo se ve suprimido por el scaldasonno e incluso el sexo se reduce a la distancia de una pantalla o la suavidad de una muñeca plástica. Lo mismo mermaría toda posibilidad de vincularse con la naturaleza o incluso con los otros humanos, deviniendo toda relación en instrumental. Por estas razones, resultaría una paradoja activar un arte cyborg, que lleva a la tecnología incluso al interior del sujeto, como un arte de resistencia crítica. En este tipo de estrategias, el humano se vería fundido y fusionado con el aparato a tal nivel, que se perderían los límites de la interfaz, trascendiendo el cuerpo a nivel del olvido. Finalmente, esto solo contribuiría a evidenciar cómo los metaprogramas de producción homogenizan completamente al sujeto, volviéndolo una maquinaria.

La sociedad que proyecta y realiza la transformación tecnológica de la naturaleza, altera la base de la dominación, reemplazando gradualmente la dependencia personal (del esclavo con su dueño, el siervo con el señor de la hacienda, el señor con el donador del feudo, etc.) por la dependencia al 'orden objetivo de las cosas' [...] (Marcuse 171).

En esta línea, sin embargo, debiéramos preguntarnos si es que existe acaso salida o estrategia posible de subversión ante un sistema que parece estar blindado por todas partes. Tal vez, la primera forma de emancipación consista en asumir esta nueva realidad global mediatizada y los cambios en nuestro cuerpo como parte de los procesos de mutación de la especie en virtud de la adaptación al medio. Por otra parte, tanto artistas como teóricos del cyborg art trabajan en la necesidad de franquear los límites impuestos para re-entender nuestra realidad en el mundo. Haraway señala: "No existe separación ontológica, fundamental en nuestro conocimiento formal de máquina y organismo, de lo técnico y lo orgánico" (cit. en De la Garza). 
$\mathrm{Al}$ asumir esta fusión, nos es posible comenzar a entender nuestras nuevas pautas identitarias: "es un ataque contra la identidad propia de occidente que consiste en la fusión de los límites y que nos puede ayudar a escapar de nuestra condición humana oprimida, mediatizada, explotada, idéntica, fija y estática, reproductora de realidades injustas. La tecnología tiene así un poder emancipador" (De la Garza). Es en el marco de esta conciencia renovadora, que la decisión, cuasi absurda, de implantarse una falsa oreja en el brazo, que además no solo recibe sonidos sino que permite reproducirlos, actúa como un acto crítico, consciente y responsable para con el propio cuerpo del artista. Se despliega en este acto el poder del artista soberano en el sentido que es capaz de trascender todo determinismo tecnológico a través de la posibilidad de lo lúdico, que subvierte los órdenes de visualidad, percepción, y modulación del cuerpo. La oreja es un órgano receptivo, asociado en la tradición occidental a una necesaria distancia -al igual que el ojo-con los fenómenos, promoviendo por tanto con fuerza el estado de diferenciación sujeto-naturaleza. En este caso, la oreja no solo está desplazada de su sitio, sino que alterada en su función, con lo que pasa de ser inerte a la producción a volverse productiva. En este sentido es interesante el que esté dispuesta en el brazo, órgano asociado al trabajo que aquí se vuelve útil en un sentido performativo, para hacer del cuerpo un sistema operativo más extenso. La oreja aparece entonces como una estructura susceptible de ser percibida desde otro lugar: "Una oreja es una estructura bella y compleja. En acupuntura, la oreja es el lugar desde donde se estimulan los órganos del cuerpo. No solo es el órgano del oído, sino también el del equilibrio. Tener una tercera oreja iría más allá del exceso anatómico y visual" (Stelarc, "Visiones parásitas. Experiencias alternantes, íntimas e involuntarias"). Surge entonces un órgano telemático más que biológico, que amplía las posibilidades sensoriales.

Acá resulta interesante detenerse a analizar la frase de Stelarc "el cuerpo está obsoleto", entendiendo que esta aseveración refiere a que tanto la percepción que tenemos sobre nuestra estructura corporal, como de sus posibilidades, deben modificarse a fin de ampliar las potencialidades de expresión y acción. Desde una cierta perspectiva, podríamos leer tanto esta frase como su acción desde una óptica profundamente dualista, donde es la mente y la voluntad del artista las que operan por sobre su cuerpo, que aparece como un cúmulo de membranas dispuestas para cualquier modificación o alteración. En la búsqueda de trascendencia física, Stelarc deja atrás el cuerpo orgánico y propone un nuevo cuerpo maquinizado e interfásico. Sin embargo, cabe la pregunta en la materialidad de la acción misma, por la posibilidad efectiva de dejar atrás el cuerpo, considerando que justamente la radicalidad de la performance está en modificar la carne, metiéndose más que nunca en ella. En efecto, volvemos con esto a una condición sine qua non de lo humano en el mundo, que es el estar delimitados y anclados en nuestra corporalidad. Las únicas muestras que han dejado huella en sus intentos de salir del cuerpo, donde podemos mencionar desde las búsquedas místicas a las chamánicas, demandan de técnicas y disciplinas marcadamente físicas, donde a través del dominio, control y luego la extenuación o quiebre del ego, se logra producir este fenómeno transcorpóreo de abandonar la matriz. Más allá de las explicaciones religiosas o científicas que existan para explicar estos casos, 
interesa resaltar este doble juego del entrar-controlar para salir-descontrolar, cuestión que en su duplicidad trasciende la lógica de las oposiciones cuerpo-alma/mente y que marca en el cuerpo el eje de su acción. En este sentido, el cuerpo no estaría obsoleto sino más presente y activo que nunca, demandando por cierto una re-actualización de su mirada.

\section{El consentimiento informado como norma en tensión}

Debiéramos entonces volver a detenernos en el tema del consentimiento informado y verificar hasta qué punto esta conciencia crítica y responsabilidad para con el propio cuerpo puede estar amparada por la bioética. Para ello, es necesario revisar algunos parámetros básicos que sustentan esta norma: "El informe Belmont identificó los principios éticos básicos a tener en cuenta en la investigación biomédica y los llamó 'de beneficio' (beneficencia-no maleficencia), 'de respeto por las personas' (autonomía) y 'de equidad' (justicia)" (La Rocca et al. 173). El primero de estos principios refiere a la obligación de obtener un máximo beneficio para el paciente en cualquier intervención médica, "pero también implica condenar todo acto que dañe deliberadamente a las personas, aun con el pretexto de un beneficio mayor (principio de no maleficencia)" (La Rocca et al. 173). El segundo principio se sustenta en que la persona tenga toda la información posible sobre el procedimiento y sus consecuencias y que sea capaz de entenderla a cabalidad. El tercer principio refiere al acceso equitativo de las personas frente a una necesidad médica.

Aquí podemos poner en duda la obra The third ear bajo parámetros bioéticos ${ }^{5}$. En primer lugar, resulta cuestionable pensar esta obra en términos de salud, ya que en ningún caso resulta benéfica para Stelarc la operación desde una perspectiva física. Él posee sus dos orejas normales y operativas, no requiere intervención alguna para mejorar ni su aspecto ni su función, menos aun implantarse una tercera. Si bien podría considerarse entonces desde la perspectiva de la cirugía estética, se filtra de todos modos la pregunta por el límite, ya que el canon de belleza en relación a la tercera oreja es del todo personal, no guarda vínculo con una estructura anatómica sana. Aquí pareciera ser que Stelarc quiere autoconvertirse en un monstruo, neutralizando esta acción bajo la de una medicina que ha adquirido el saber, la competencia y el privilegio de configurar, modificar y ajustar el cuerpo trastocando los parámetros ético-estéticos de bienestar y belleza por anomalías que provienen muchas veces de áreas externas a la salud tales como la publicidad o la moda. ¿Es que ponerse una oreja en el brazo es más feo o menos saludable que un par de labios hiperhinchados o traseros gigantes de metacril?

En segundo lugar, y siguiendo la lógica de la autonomía, vemos que en este ejercicio de apropiación del cuerpo, Stelarc extrema la figura del consentimiento informado, tradicionalmente aplicada a través de un protocolo que cede los derechos de un paciente

5 Como dato anecdótico se puede agregar que el procedimiento quirúrgico necesario para la obra fue rechazado por varios médicos y que de hecho se consideró ilegal en EEUU. 
a un médico con mayor conocimiento. Al trabajar en conjunto con el equipo científico, el artista opera más que solo informándose con respecto a las posibles consecuencias de su accionar, sino que en absoluto otorga a un tercero el derecho a decidir sobre su cuerpo. (En este caso, este tercero operando como la ciencia médica o la misma sociedad con sus patrones sobre lo que es bello, sano o útil). Es Stelarc quien juega con los cánones y los límites, asumiendo los riesgos implícitos en este acto, con plena autonomía de su derecho a decidir. La cuestión de peso aquí no está solo en “informarse para delegar”, sino asumir un rol de participación implícita en el proceso científico que opera sobre el cuerpo.

En tercer lugar, y en lo referente al problema de la equidad, si bien The third ear no limita el acceso a otras personas a los servicios médicos o tecnológicos, si pareciera burlarse con esta acción de aquellos en condiciones vulnerables. La obra que analizamos, absurda desde la perspectiva de la necesidad, parece por tanto una bofetada en la cara para "quienes no tienen posibilidades de acceder no solo a las nuevas tecnologías, altamente sofisticadas y de elevado costo, sino que ni siquiera a las formas tradicionales y básicas del sistema sanitario" (La Rocca et al. 172). Recordemos que uno de los objetivos de la obra es su exposición y metaconexión (al poseer un micrófono y antena wi-fi) y busca, por las mismas razones, que la gente interactúe con la obra a nivel mundial. En este sentido, esta acción está constantemente a disposición de todo individuo haciendo evidente el espectáculo mercantil de este monstruo que puede, por sus nuevas capacidades sobrehumanas, transformarse en un solo paso en un ídolo que figuraría como inalcanzable a no ser de contar con los recursos económicos para imitarlo. Lo que resulta interesante aquí, sin embargo, es como nuevamente se revela la posibilidad de generar una nueva belleza a partir de la ruptura de estructuras hegemónicas y perturbación primera de los sentidos, que se renuevan necesariamente en la dialéctica exposición-contemplación-participación que propone esta obra.

En último término, y siguiendo la pregunta por el bienestar, cabe instalar la posibilidad de que Stelarc decidiera amputarse una de sus orejas basado en el mismo fundamento artístico. ¿Debiera la medicina tolerar este tipo de acciones? ¿Es que ponerse o quitarse una oreja no es un acto deliberado de daño de una persona con respecto a sí misma? Claramente queda en evidencia con esta acción que hace falta un diálogo transdisciplinario entre artes y ciencia, entre estética y bioética, pues realizar un acto de esta radicalidad con el fin de cuestionar a la sociedad, si bien logra su cometido, puede transgredir incluso los mismos conceptos en los que todo diálogo puede sustentarse. Resulta fundamental comprender la actividad artística desde su función social renovadora, en especial desde su capacidad de anticipar y subvertir mecanismos de control como los tecnológicos. Solo actualizando los vínculos entre arte y sociedad es que podremos realizar una auténtica mirada crítica a los procesos de cambio que nuestro cuerpo y especie viven en la actualidad. 


\section{Referencias}

Bajtín, Mijaíl. Hacia una filosofía del acto ético. San Juan: Universidad de Puerto Rico, 1997. Medio impreso.

Ciénaga, Érika. Mutaciones del cuerpo: hacia la construcción de nuevas formas de subjetivación y sus implicaciones éticas. Reflexiones en torno a lo abyecto en las prácticas estéticas contemporáneas. Iztacala. <http://www.iztacala.unam.mx/errancia/v1/ PDFS_1/POLIETICAS1_MUTACIONESDELCUERPO.pdf $>$. Fecha de ingreso: marzo 2013. Sitio web.

Cimaomo, Gabriel. Más allá de la acción artística. Una mirada sobre el cuerpo pulsional en el arte contemporáneo. Kaleidoscopio. $<$ http://www.kaleidoscopio.com.ar/fs_files/ user_img/Arte/M\%C3\%A1s\%20all\%C3\%A1\%20de\%20la\%20acci\%C3\%B3n\%20 art\%C3\%ADstica.pdf>. Fecha de ingreso: marzo 2013. Sitio web.

Cotaimich, Valeria. "Hacia un teatro de cyborgs. Artes escénicas, tecnología/s y subjetividad/es” ICONO 1410 (2008). <http://www.icono14.net/ojs/index.php/icono14/ article/view/365>. Fecha de ingreso: marzo 2013. Sitio web.

De la Garza, Ma. Teresa. Cuerpo y sociedades tecnocientificas. Proteo: diálogos de ética y bioética. <http://www.dialogos.unam.mx/mtdelagarza.pdf>. Fecha de ingreso: marzo 2013. Sitio web.

Foucault, Michel. El nacimiento de la clínica, una arqueología de la mirada médica. Buenos Aires: Siglo XXI editores, 2001. Medio impreso.

La Rocca, Susana, Gladys Martínez, Alejandra Rascio y Mirta Bajardi. "La investigación biomédica y el consentimiento informado en el ámbito de las poblaciones e individuos vulnerables". Acta Bioethica 11(2005). 169-81. Medio impreso.

Larratt, Shannon. ModCon: The Secret World of Extreme Body Modification. Canada: BME books, 2002. Medio impreso.

Le Breton, David. Adiós al cuerpo. Colonia del Valle: la Cifra Editorial, 2007. Medio impreso.

Maliandi, Ricardo, Ética: conceptos y problemas. Buenos Aires: Ed. Biblos, 1994. Medio impreso.

Manzini, J.L y Salvador, H.. La autonomía del sujeto y el consentimiento informado. Elemental análisis histórico y conceptual. Estado actual y perspectivas en la República Argentina. Buenos Aires: Quirón, 1993. Medio impreso.

Marcuse, Herbert. El hombre unidimensional. Buenos Aires: Planeta, 1993. Medio impreso.

Stelarc. "Visiones parásitas. Experiencias alternantes, íntimas e involuntarias". Revista Mecad Electronic Journal 1. <http://ejournalmecad.files.wordpress.com/2008/06/ ejournal1.pdf $>$. Fecha de ingreso: mayo 2013. Sitio web.

--- "Ear on Arm”. Stelarc. <http://stelarc.org/?catID=20242>. Fecha de ingreso: junio 2013. Sitio web.

Stiegler, Bernard. La Técnica y el Tiempo. Vol. 1. Hondarrabia: Ed. Hiru, 2002. Medio impreso. Villarroel, Raúl. “Bioética Hermenéutica”. Acta Bioethica 1 (2000). 143-57. Medio impreso. 